\title{
Measurement and Analysis of Brain Deformation During Neurosurgery
}

\author{
T. Hartkens, D. L. G. Hill*, A. D. Castellano-Smith, D. J. Hawkes, C. R. Maurer, Jr., A. J. Martin, W. A. Hall, \\ H. Liu, and C. L. Truwit
}

\begin{abstract}
Recent studies have shown that the surface of the brain is deformed by up to $20 \mathrm{~mm}$ after the skull is opened during neurosurgery, which could lead to substantial error in commercial image-guided surgery systems. We quantitatively analyze the intraoperative brain deformation of 24 subjects to investigate whether simple rules can describe or predict the deformation. Interventional magnetic resonance images acquired at the start and end of the procedure are registered nonrigidly to obtain deformation values throughout the brain. Deformation patterns are investigated quantitatively with respect to the location and magnitude of deformation, and to the distribution and principal direction of the displacements. We also measure the volume change of the lateral ventricles by manual segmentation.

Our study indicates that brain shift occurs predominantly in the hemisphere ipsi-lateral to the craniotomy, and that there is more brain deformation during resection procedures than during biopsy or functional procedures. However, the brain deformation patterns are extremely complex in this group of subjects. This paper quantitatively demonstrates that brain deformation occurs not only at the surface, but also in deeper brain structure, and that the principal direction of displacement does not always correspond with the direction of gravity.

Therefore, simple computational algorithms that utilize limited intraoperative information (e.g., brain surface shift) will not always accurately predict brain deformation at the lesion.
\end{abstract}

Index Terms-Interventional MRI, intraoperative brain deformation, nonrigid image registration.

\section{INTRODUCTION}

I MAGE-GUIDED surgery (IGS) systems register preoperative tomographic images to the intraoperative coordinate system of the patient. The resulting transformation or mapping is used to display the position and orientation of tracked surgical instruments on reformatted image slices and renderings of

Manuscript received October 22, 2001; revised August 28, 2002. The work of T. Hartkens and A. D. Castellano-Smith was supported by the UK Engineering and Physical Sciences Research Council. The Associate Editor responsible for coordinating the review of this paper and recommending its publication was J. Duncan. Asterisk indicates corresponding author.

T. Hartkens, A. D. Castellano-Smith, and D. J. Hawkes are with the Computational Imaging Science Group, Guy's Hospital, King's College London, London SE1 9RT, U.K.

*D. L. G. Hill is with the Computational Imaging Science Group, Guy's Hospital, King's College London, London SE1 9RT, U.K. (e-mail: Derek.Hill@kcl.ac.uk)

C. R. Maurer, Jr. was with the King's College London, London SE1 9RT, U.K. He is now with the Department of Neurosurgery, Stanford University, Stanford, CA 94305 USA.

A. J. Martin was with the University of Minnesota, Minneapolis, MN 55455 USA. He is now with the Department of Radiology, University of California, San Francisco, CA 94143 USA.

W. A. Hall, H. Liu, and C. L. Truwit are with the Departments of Radiology and Surgery, University of Minnesota, Minneapolis, MN 55455 USA.

Digital Object Identifier 10.1109/TMI.2002.806596 the brain and anatomical structures of interest. In neurosurgery, commercial IGS systems make the assumption that a patient's head and brain is a rigid body. Recent studies have shown that this assumption is not valid and report on significant brain deformation (brain shift) after the skull is opened and before the interventional procedure is started, e.g., [1]-[6]. If the tissue deformation is large relative to the amount of surgical accuracy required, then the overall accuracy of the IGS system will be substantially reduced.

Several approaches have been developed to address the brain deformation problem. A magnetic resonance (MR) scanner that has been modified for intraoperative surgery can be used to scan the patient multiple times during the intervention and monitor the brain deformation (e.g., [7]). Alternatively, real-time ultrasound systems have been proposed to correct for the brain deformation [8], [9]. Although these devices can provide surgeons with updated images during the intervention, the use of preoperative data is still of clinical interest. For instance, it may be desirable to have images from other modalities (e.g., positron emission tomography, functional MR imaging) and preoperatively prepared data (e.g., segmentations of clinically important anatomical structures) displayed in an IGS system. In particular, the fusion of these data with intraoperatively acquired images provides surgeons with additional information. Furthermore, since interventional MR scanners are expensive, complicate access to the patient and prevent the use of standard metallic surgical instruments because of their high magnetic fields, their usage is restricted and for some cases it may be preferable to use preoperatively acquired images.

Recent studies develop biomechanical models which estimate displacements in order to update the preoperative images [10]-[15]. These models are based on physical brain deformation and require intraoperative measurements to constrain their model. In general, these studies assume, that the brain deformation can be estimated by introducing simple physical models for the cause of deformation, e.g., direction of gravity or size of the resection [14], [15].

In this paper, we qualitatively and quantitatively investigate brain deformation based on MR images from 24 patients that underwent cranial neurosurgical procedures at the University of Minnesota. The images were acquired at the start and end of each procedure using an interventional MR scanner in the operating room. The aim of this paper is to investigate the pattern of brain deformation with respect to location, magnitude, and direction and to consider the implications of this pattern on models for correcting brain deformation or on the errors in IGS systems due to brain deformation. We focus on three questions: 1) Where 
is the deformation, e.g., does it occur only at the brain surface or also in deeper brain structures? 2) What is the magnitude of the deformation? 3) Can simple rules describe or predict the deformation, e.g., loss of cerebrospinal fluid (CSF) causes the brain to sink in direction of gravity?

The volume change in each lateral ventricle is calculated after manually segmenting the lateral ventricles in the start- and endprocedure images. The start-procedure image is automatically registered to the end-procedure image using a nonrigid registration algorithm. The resulting transformation is a deformation vector field, which is used to quantify deformation between the images. Since the nonrigid transformation defines a displacement vector for each point in the image, in contrast to the manually determined ventricular volume changes, this approach provides deformation measurements throughout the entire brain. The deformation field is investigated quantitatively with respect to the location of the deformation, the magnitude of deformation in relation to the distance to the brain surface, the principal direction of the deformation, and the distribution of the displacement direction.

Studies prior to the mid-1990s mentioned brain shift (e.g., [16]), but did not quantify the deformation. More recent studies determined either the deformation at the brain surface, [2]-[4], [17], or the volume change of manually segmented anatomical structures [1], [5], [6], [17]. While these studies reported manual and/or local measurements of the deformation at certain surfaces or structures during neurosurgery, we are interested in an approach that provides deformation values throughout the entire brain. A similar approach has already been used to quantify the volume change of anatomical structures in the developing brain of children [18] and of the temporal lobe of Alzheimer's disease patients [19], [20]. Hata et al. [21] (see, also, [22] and [23]) applied a similar method to measure brain deformation during neurosurgery, but did not apply any complex operators to the deformation field, did not consider the location of deformation, and did not analyze volume changes of specific anatomical structures.

The paper is organized as follows: Section II describes the MR image acquisition parameters and methods we used to quantify and localize the brain deformation. Section III presents the results of the quantitative measurements, presents a visual assessment of the deformation, and provides illustrations of the deformation fields.

\section{MATERIALS AND MeTHODS}

This section describes the image acquisition and the methods we used to quantify and localize the brain deformation. First, the volume change of the lateral ventricles is determined by manual segmentation in the start-procedure and end-procedure image. Then, in order to investigate the deformation throughout the whole brain the start- and end-procedure images are registered nonrigidly and the resulting deformation vector field is analyzed with respect to the magnitude, direction, and location of displacement. At the end of this section the deformation localization methods are described.
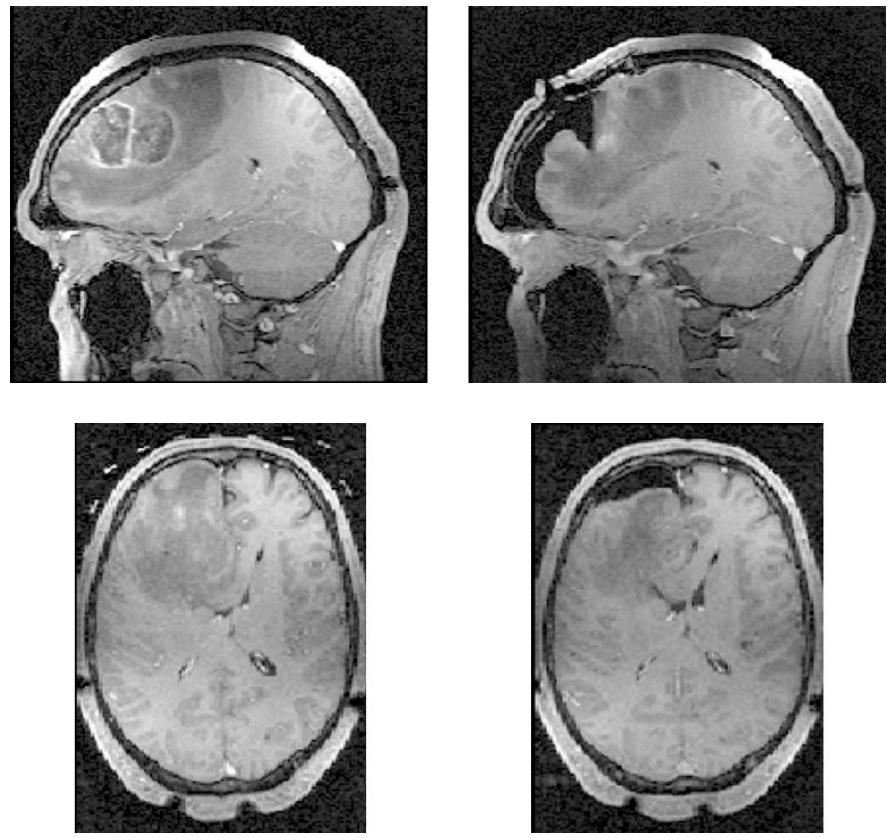

Fig. 1. Example interventional start-procedure (left) and end-procedure (right) image (case resection8). (top) Sagittal plane (as acquired) and (bottom) reformatted transaxial plane.

\section{A. Image Acquisition}

This paper is based on MR images from 24 patients that underwent cranial neurosurgical procedures in the interventional MR suite at the University of Minnesota. During surgery, magnetization-prepared rapid gradient echo (MP-RAGE) volume images were acquired with a 1.5-T interventional MR scanner (Philips ACS-NT, Philips Medical Systems). In addition to the normally acquired images, which generally contain only a small number of slices, additional whole brain images volumes were obtained at the start and at the end of each intervention (see Fig. 1). The voxel dimensions are typically $0.9 \times 0.9$ $\times 1.5 \mathrm{~mm}$, and the readout gradient is oriented in the cranial-caudal direction with a magnitude of $4.7 \mathrm{mT} / \mathrm{m}$. Since the images were acquired using an open-style radio-frequency coil (a "synergy" phased array coil), some images have substantial nonuniform signal intensity. Table I summarizes details of the 24 cases studied in this investigation.

Gradient echo images have high resolution (small pixel size), and the relatively high readout-gradient magnitude $(4.7 \mathrm{mT} / \mathrm{m})$ used in this study produces images with relatively little geometrical distortion in the read-out direction due to static field inhomogeneity [24]. However, gradient echo images are sensitive to magnetic susceptibility differences in the object being imaged and, consequently, there can be signal loss at the boundary between air and soft tissue. Spin echo images do not have this potential problem of signal loss. For patients undergoing resections, the brain is exposed to the air and air can enter the head. The magnitude of any brain deformation could, therefore, be exaggerated by signal loss due to susceptibility artifacts. To establish the extent of this problem, we also acquired T2-weighted turbo spin echo images from some patients. 
TABLE I

DETAILS OF 24 CASES STUdied USING INTERVENTIONAL MR. IN THE CASES Marked With ${ }^{\dagger}$, THE SeCONd IMAGe Was ACQuired IN THE MidDle OF THE INTERVENTION AND NOT AFTER THE INTERVENTION

\begin{tabular}{c|r|l|l|l}
\hline Case & Age & Gender & Lesion position & Lesion type \\
\hline \hline resection1 & 53 & male & left occipital & lung metastasis \\
\hline resection2 & 5 & male & left occipital & astrocytoma with necrosis \\
\hline resection3 & 69 & male & left frontal & glioblastoma multiforme \\
\hline resection4 & 3 & female & right medio temporal & low grade glioma \\
\hline resection5 & 39 & female & right occipito parietal & meningioma \\
\hline resection6 & 41 & female & right frontal & glioma \\
\hline resection7 & 36 & female & superficial left temporal & glioma \\
\hline resection8 & 57 & male & left frontal & glioblastoma multiforme \\
\hline resection9 & 57 & male & left temporal & glioblastoma multiforme \\
\hline resection10 & 26 & female & left temporal & astrocytoma \\
\hline resection11 & 34 & male & right medio frontal lesion & low grade glioma \\
\hline resection12 & 60 & male & left temporal & glioblastoma multiforme \\
\hline resection13 & 54 & male & occipital & glioblastoma multiforme \\
\hline \hline biopsy1 & 38 & female & left frontal lesion & oligodendroglioma \\
\hline biopsy2 & 72 & female & left parietal & glioblastoma multiforme \\
\hline biopsy3 & 47 & female & left temporal parietal & glioblastoma multiforme \\
\hline biopsy4 & 25 & female & left parietal & astrocytoma \\
\hline biopsy5 & 73 & male & left frontal & glioma \\
\hline \hline functional1 & 50 & female & bilateral & obsessive compulsive disorder \\
\hline functional2 & 43 & male & right temporal & brain damage and tremor \\
\hline functional3 ${ }^{\dagger}$ & 58 & male & left & tremor \\
\hline functional4 & 61 & male & left & tremor \\
\hline functional5 & 84 & female & left & tremor \\
\hline functional6 & 61 & male & right & tremor \\
\hline & & & &
\end{tabular}

\section{B. Segmentation of the Lateral Ventricles}

In order to estimate brain deformation in deeper brain structures during the surgical procedure, we manually segment the lateral ventricles in the start- and end-procedure images using Analyze (Mayo Clinic). Based on these segmentations, volume changes were calculated separately for the lesion's ipsi- and contra-lateral ventricles, and for both ventricles together.

\section{Calculation of the Deformation Vector Field}

While manual segmentation provides the volume change of certain structures, nonrigid registration of the start- and endprocedure images produces displacement values throughout the brain for each voxel in the image. The resulting displacement map can be used to investigate deformation in the entire brain and to detect typical deformation patterns.

First, we rigidly register images to correct for any rigid-body motion between the start- and end-procedure images by maximizing the normalized mutual information (NMI) in the joint probability distribution between the two images [25]. Then we nonrigidly register images by deforming a regular grid of control points in the start-procedure image [26]. The nonrigid registration algorithm moves the control points; tissue motion is described by free-form deformation using B-spline approximation between the control points. The control point spacing determines the flexibility of the grid and was set in our investigation by $15 \mathrm{~mm}$. Again, NMI is used as a measure of the similarity of the images. This algorithm was previously evaluated for the registration of three-dimensional (3-D) breast MR images [27] and of 3-D brain MR images [28]. In the latter study, the displacement vectors determined by the nonrigid algorithm were compared with the displacements of manually determined point landmarks in deformed brain MR image. It was shown that $68 \%$ of the values of the displacement map are within $0.5 \mathrm{~mm}$ of the

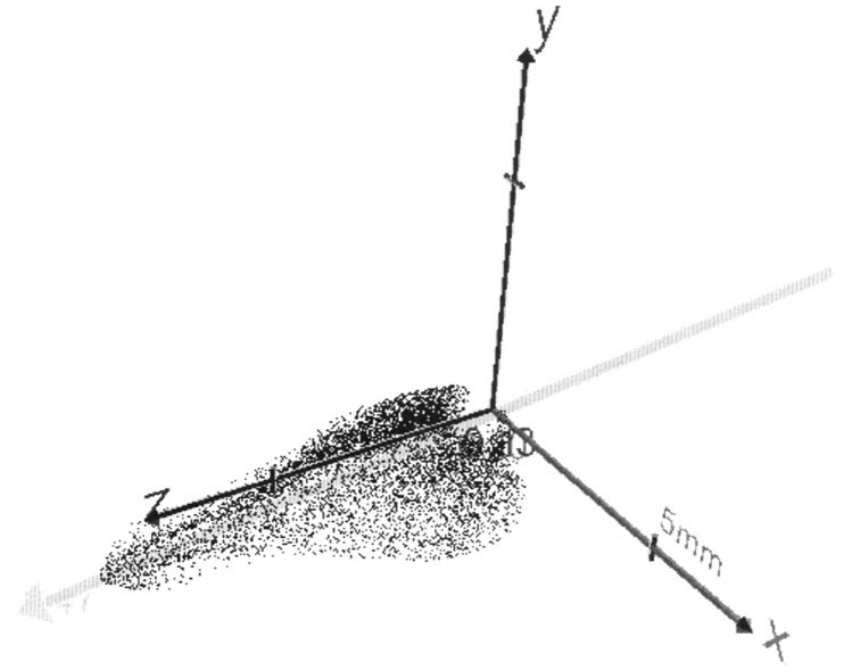

Fig. 2. Distribution of the displacement vectors of case resection 7 . The displacement vectors are visualized as points in the 3-D coordinate system whereby the axes represents the components of the vectors. Displacement vectors with a magnitude of less than $1 \mathrm{~mm}$ are not shown in this diagram. The large vector in the diagram represents the first axis of the PCA. The variance in direction of this vector is a measure for the magnitude of the displacement field. The other two axis of the PCA are visualized in relationship to the variance of first axis. Because the variance in their direction is very low in comparison to the first axis, they can hardly be seen in this visualization.

interactively measured values and $90 \%$ are within $1.0 \mathrm{~mm}$. Since the reproducibility error of the interactive measurements was up to $0.9 \mathrm{~mm}$, this suggests that the results of the automatic nonrigid registration algorithm agree with the interactive measurements to the precision of these.

The registration algorithm results in a 3-D deformation field $D$ which is mathematically defined as a vector field $D: R^{3} \rightarrow$ $R^{3}$ and maps a point $\mathbf{p}_{\text {source }}(x, y, z)$ from the source image to the target image $\mathbf{p}_{\text {target }}=D\left(\mathbf{p}_{\text {source }}\right)$.

Displacement Vectors: Usually the deformation field is visualized as a displacement field $d$ (see, e.g., Fig. 6). This field represents the displacement of each point and is defined on the basis of the deformation field: $D(\mathbf{p})=\mathbf{p}+d(\mathbf{p})$.

We are not only interested in the magnitude of the displacement vectors, but also in the distribution of their directions and in the main direction of the whole displacement field. In order to analyze the displacement directions, principal component analysis (PCA) is applied on the displacement field in the following way: the displacement vectors are considered as points in a 3-D coordinate system, whereby the axes of the coordinate system represent the components of the displacement vectors (see Fig. 2). Then, a vector is adjusted through the point cloud in such way that the variance of the points is maximal along the vector. In contrast to the classical PCA, the vector is determined with respect to the origin of the diagram and not with respect to the mean of the points. This vector can be considered as the principal direction of the displacement field and the variance in its direction as a measure for the over-all magnitude of the deformation. Another vector perpendicular to the first is determined that maximizes the variance of the points in its direction again. A third vector is perpendicular to the first and second axis, and the variance in this direction is calculated. Usually, the variances in the direction of these three vectors 

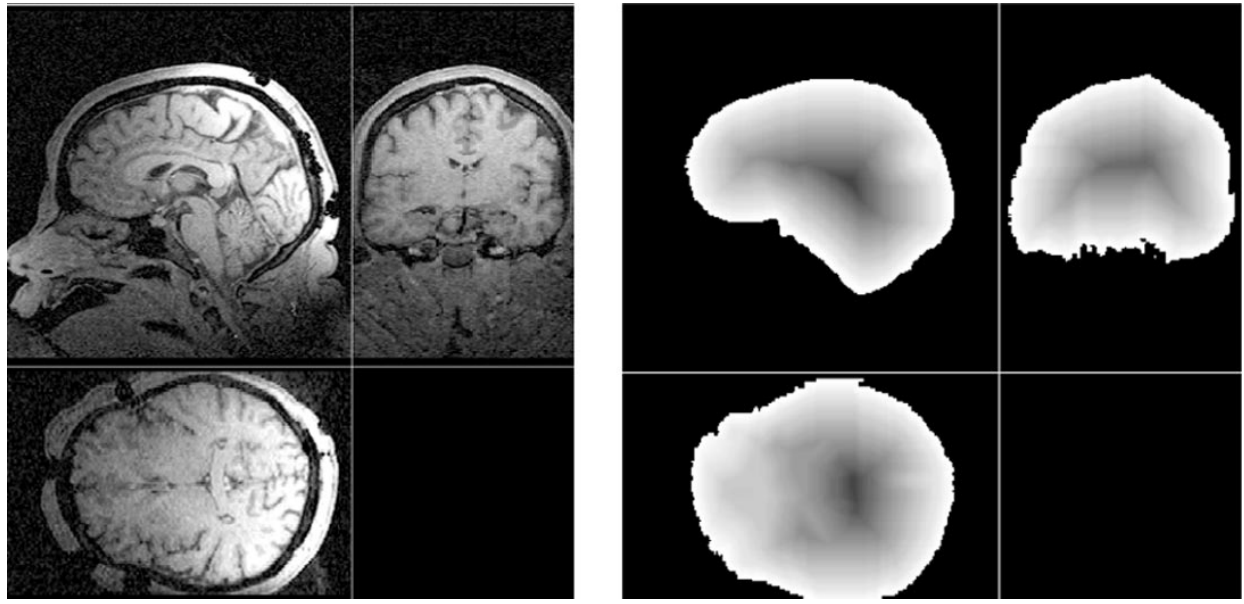

Fig. 3. Distance map for resection5. (left) End-procedure image and (right) corresponding distance map. The gray values inside the brain represent the distance to the brain surface (darker values correspond to larger distance).
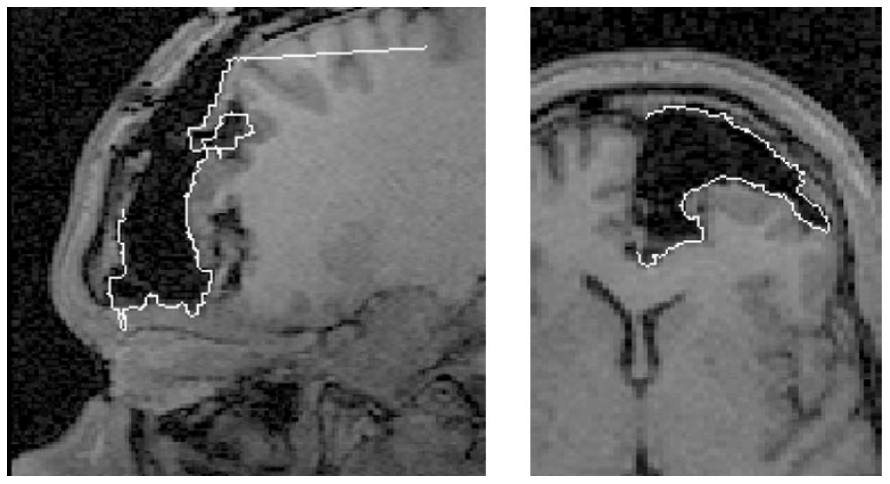

Fig. 4. Example sagittal and coronal slices from resection11. The brain surface contour of the spin-echo image is overlaid on the gradient-echo image. The spin-echo image were taken 3 min before the gradient-echo image. The good agreement between contours suggests that signal loss due to susceptibility difference at the air/tissue interface is not a cause of large errors.

are multiplied to measure the distribution of the point cloud or displacement directions, respectively (this corresponds to the so-called volume of the covariance ellipsoid).

Jacobian Operator: The Jacobian operator can be used to measure local relative tissue volume change throughout the brain. It is defined at the point $P$ as the determinant of the Jacobian matrix of the deformation field

$$
J a c_{\mathbf{p}}(D)=\operatorname{det}\left(\nabla_{\mathbf{p}} D\right)=\left|\begin{array}{lll}
\frac{\partial D_{x}}{\partial x} & \frac{\partial D_{x}}{\partial y} & \frac{\partial D_{x}}{\partial z} \\
\frac{\partial D_{y}}{\partial x} & \frac{\partial D_{y}}{\partial y} & \frac{\partial D_{y}}{\partial z} \\
\frac{\partial D_{z}}{\partial x} & \frac{\partial D_{z}}{\partial y} & \frac{\partial D_{z}}{\partial z}
\end{array}\right| .
$$

The Jacobian operator relates an elementary volume $\delta V_{\text {source }}$ in the source image to the corresponding deformed volume $\delta V_{\text {target }}$ in the target image

$$
\delta V_{\text {target }}=J a c_{\mathbf{p}}(D) \cdot \delta V_{\text {source }}
$$

and can be interpreted as the local relative change of an elementary volume [29], [30]. Thus, operator responses larger than one can be considered as a local expansion of the volume and responses less than one as a local shrinking of the volume at point $\mathbf{p}$. The response $J_{a c_{\mathbf{p}}}(D)=1$ means that the volume is preserved locally at this point. We apply the Jacobian operator to the deformation field determined by the nonrigid algorithm to analyze the local volume change for each point in the image.

\section{Localization of Brain Deformation}

In addition to the magnitude of deformation, we are also interested in the location of the deformation and whether a typical pattern can be found in their spatial distribution. First, we separately analyze the values in the ipsi-lateral and in the contra-lateral hemisphere to the lesion. Second, a distance map is used, which specifies for each voxel inside the brain its distance to the brain surface.

Separating Ipsi- and Contra-Lateral Hemisphere: In order to investigate the deformation measurements for each hemisphere separately, the brain is segmented into the left and right hemispheres. We use the segmentation of the brain atlas Internet Brain Segmentation Repository [31] and perform an affine registration of the reference MR image of the atlas to the end-procedure images. The resulting affine transformation is used to transform the atlas segmentation to the coordinate system of the end-procedure image. Since we are not interested in the exact boundary of the brain, but only in the deformation values inside each hemisphere, the approximate segmentation obtained with this method is sufficiently accurate for our investigation.

Distance to the Brain Surface: In order to quantify the deformation with respect to the distance to the brain surface, a distance map is calculated for each patient [32]. The brain is segmented in the end-procedure images, and, starting with the voxels at the brain surface, the voxels are marked with a value that represents the distance to the surface. The result is an image in which the value of a voxel inside brain represents the distance to the brain surface (see Fig. 3). This image is used to analyze the deformation measurements with respect to distance by considering for each distance value only those deformation measurements corresponding to voxels in the distance map with that distance value. 

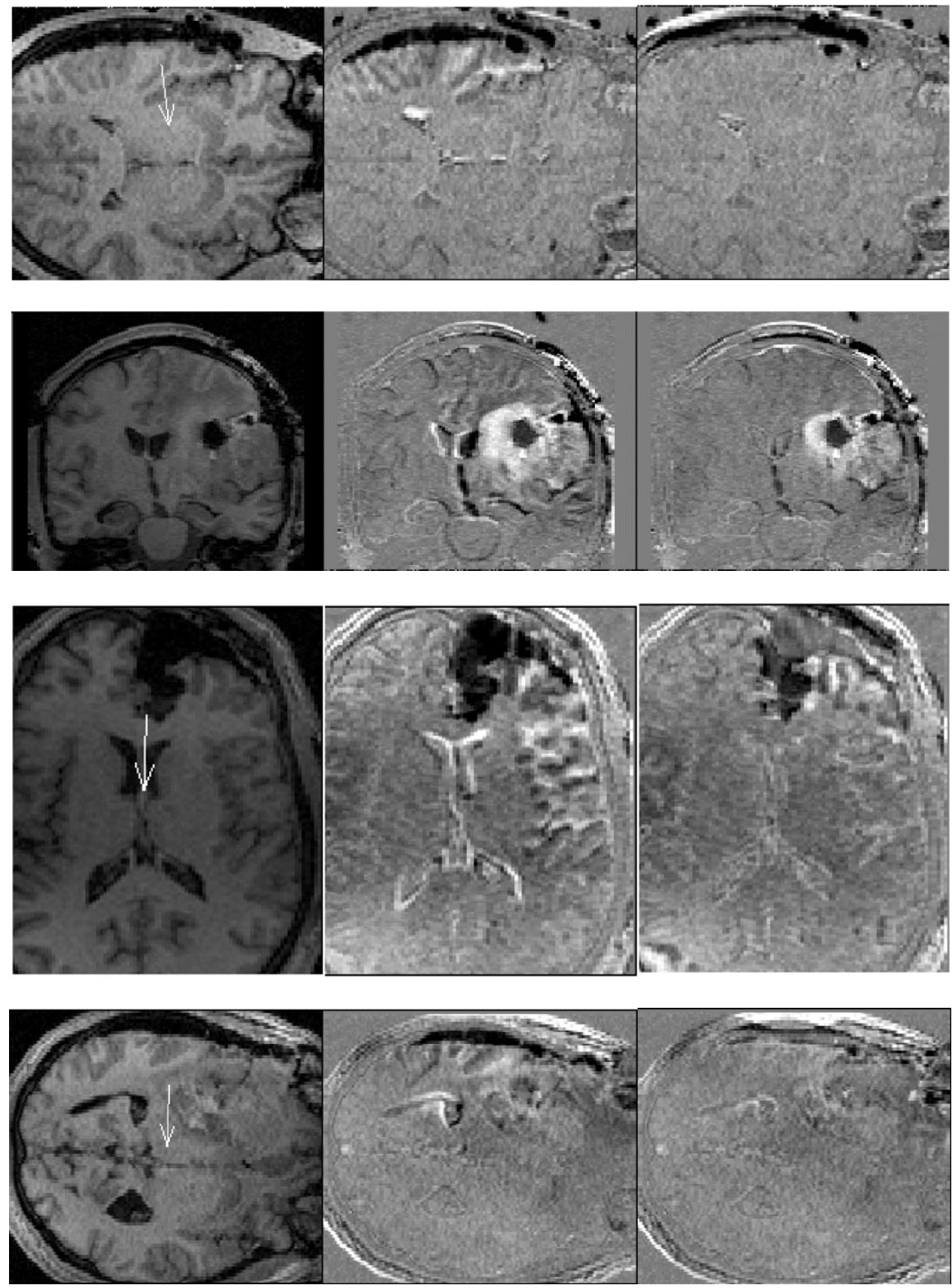

Fig. 5. Four example resection cases (from top to bottom) resection7, resection10, resection11, and resection12. The first column shows the end-procedure image for each case. The arrow in these images represents the projection of gravity direction onto the image plane. For resection 10 the gravity vectors shows out of the image plane and, therefore, is not visualized. The subtraction of the end- and start-procedure image is shown in the middle column after rigid registration and in the right column after nonrigid registration.

\section{RESULTS}

First, we compare the spin-echo images with the gradient-echo image to assess the signal loss at the boundary between air and soft tissue in the gradient-echo image which could exaggerate the magnitude of brain deformation. Fig. 4 shows the contour of the spin-echo image overlaid on the gradient-echo image. In three patients, we carried out visual assessment of the gradient echo MR volume with the brain boundary from spin echo overlaid. For these three cases, the boundaries were judged on average $2.1( \pm 1.8) \mathrm{mm}, 0.4( \pm 0.5)$ $\mathrm{mm}$, and $1.4( \pm 0.7) \mathrm{mm}$ of one another, respectively. Given the difference in read-out gradient strength between gradient-echo and spin-echo imaging, the time delay between acquisitions (between 3 and $6 \mathrm{~min}$ ), and a partial volume effect due to different slice thicknesses, these are consistent with the gradient echo and spin echo images providing equivalent information about the deformation. Thus, signal loss in the gradient echo images at the air-tissue boundary is not likely to be a major cause of error in this study.

\section{A. Visual Assessment of Brain Deformation}

The size and location of the lesion varies significantly for the cases investigated and, therefore, the comparison of the start- 

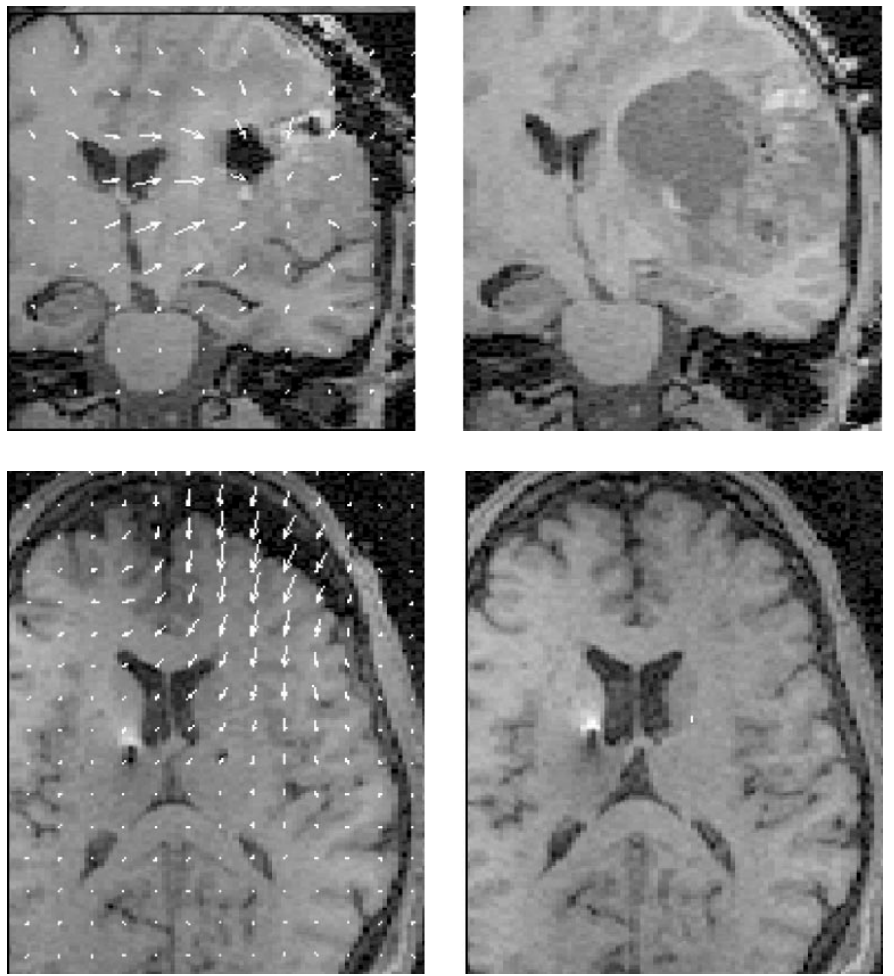

Fig. 6. Start- and end-procedure image and displacement vectors. Each subfigure shows (left) the end-procedure image and (right) the start-procedure image. The end-procedure image is overlaid with the displacement vectors determined by the nonrigid registration (rigid part of the transformation is ignored in the displacement field); (top) resection10 and (bottom) functional16.

and end-procedure image shows different types of deformation. In Fig. 5, four example resection cases and their corresponding subtraction images after rigid and nonrigid registration are depicted. Case resection7 (first row in Fig. 5) underwent a small resection in the left temporal lobe and the subtraction image after rigid registration (second column) mainly shows deformation at the surface of the brain while the contra lateral hemisphere is hardly deformed. In contrast, a deep lesion was resected in case resection10 which caused a considerable deformation of the ventricular system. Even though the deformations at the contra lateral ventricle are much less in comparison to the ipsi lateral ventricle, the brain also deforms substantially in the contra-lateral hemisphere. The subtraction image of this case suggests that there is less deformation at the brain surface than at the ventricular system. The resection of the lesion at the frontal lobe in the case resection11 caused both a brain shift at the brain surface and a deformation of deeper brain structures (see lateral ventricle). The lesion of case resection 12 was located in the left temporal lobe and the image shows similar deformation pattern to resection7. In all of these cases except in case resection 10, the subtraction image suggests that the main direction of the brain shift is in the direction of gravity (indicated by an arrow in the end-procedure images in the first column). We will see in the next section, where we analyze the distribution of the displacement vectors, that this result may not apply to all cases.

The subtraction image after applying the nonrigid transformation (third column in Fig. 5) shows that the nonrigid registration deforms the start-procedure image in such a way that it corresponds well with the deformation in the end-procedure image.
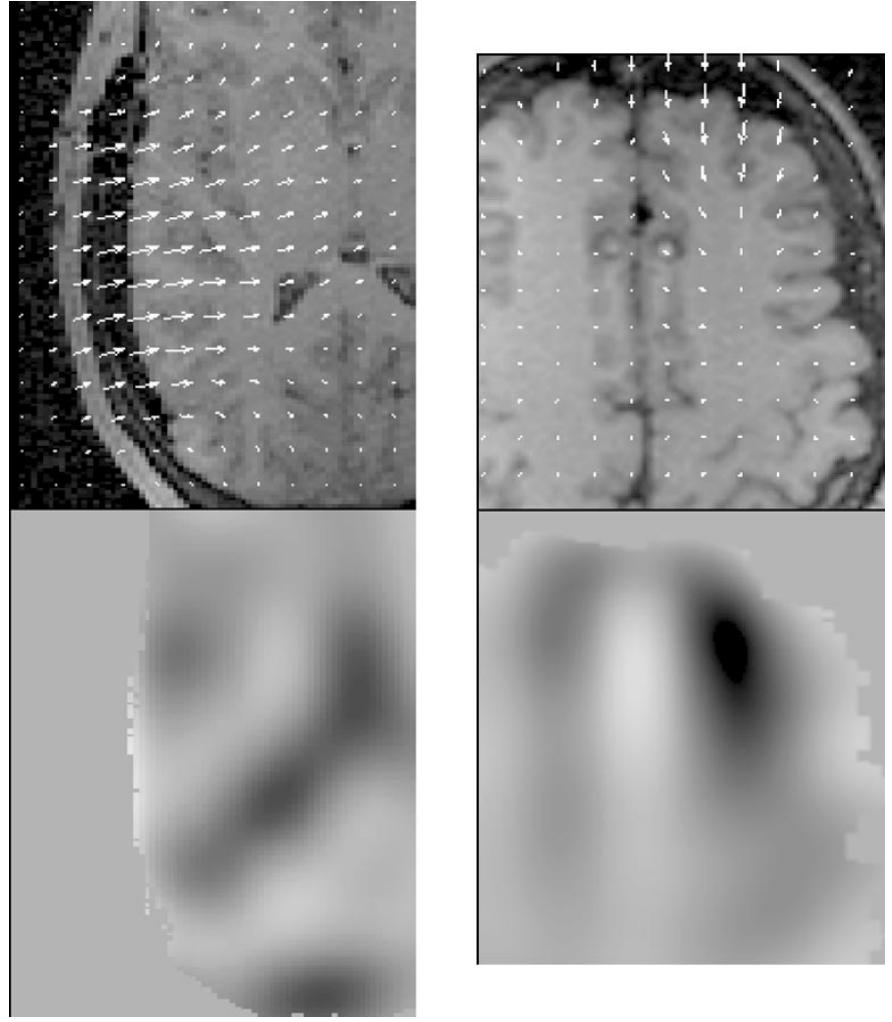

Fig. 7. Displacement vectors and volume change per voxel. The upper image in each figure shows the end-procedure image overlaid by the displacement vectors. The bottom images show the response of the Jacobian operator applied on the displacement field inside the brain. Darker gray values in the image represent a shrinking while brighter gray values represent an expansion. A medium value (similar to background) represents no volume change; (left) resection7 and (right) functional11.

The deformation map overlaid on the end-procedure image (see Fig. 6) indicates that the displacement is greatest close to the lesion or craniotomy, but can vary considerably. For instance, while the deformation in case functional6 is greatest immediately beneath the craniotomy, and near the mid-line has diminished to virtually zero, case resection 10 also shows significant displacements in the contra-lateral hemisphere.

The Jacobian operator is applied in Fig. 7 on the displacement field to visualize the relative volume change throughout the brain. Dark regions in these images indicate a shrinking of the brain and bright regions indicate an expansion. Comparing the displacement field with the Jacobian operator responses, demonstrates that a large displacement at a given position does not yield a significant volume change at that position. For example, in case resection 7 in Fig. 7 the largest displacement is at the surface while a significant volume change appears in deeper brain structures. In case functionall, significant displacement vectors are spread over the frontal lobe while the larger volume change is concentrated in a smaller region.

\section{B. Deformation in the Ipsi- and Contra-Lateral Hemisphere to the Procedure}

The deformation values in the ipsi- and contra-lateral hemisphere are considered separately for each case using the segmentation yielded by registering the brain atlas to the end-procedure 

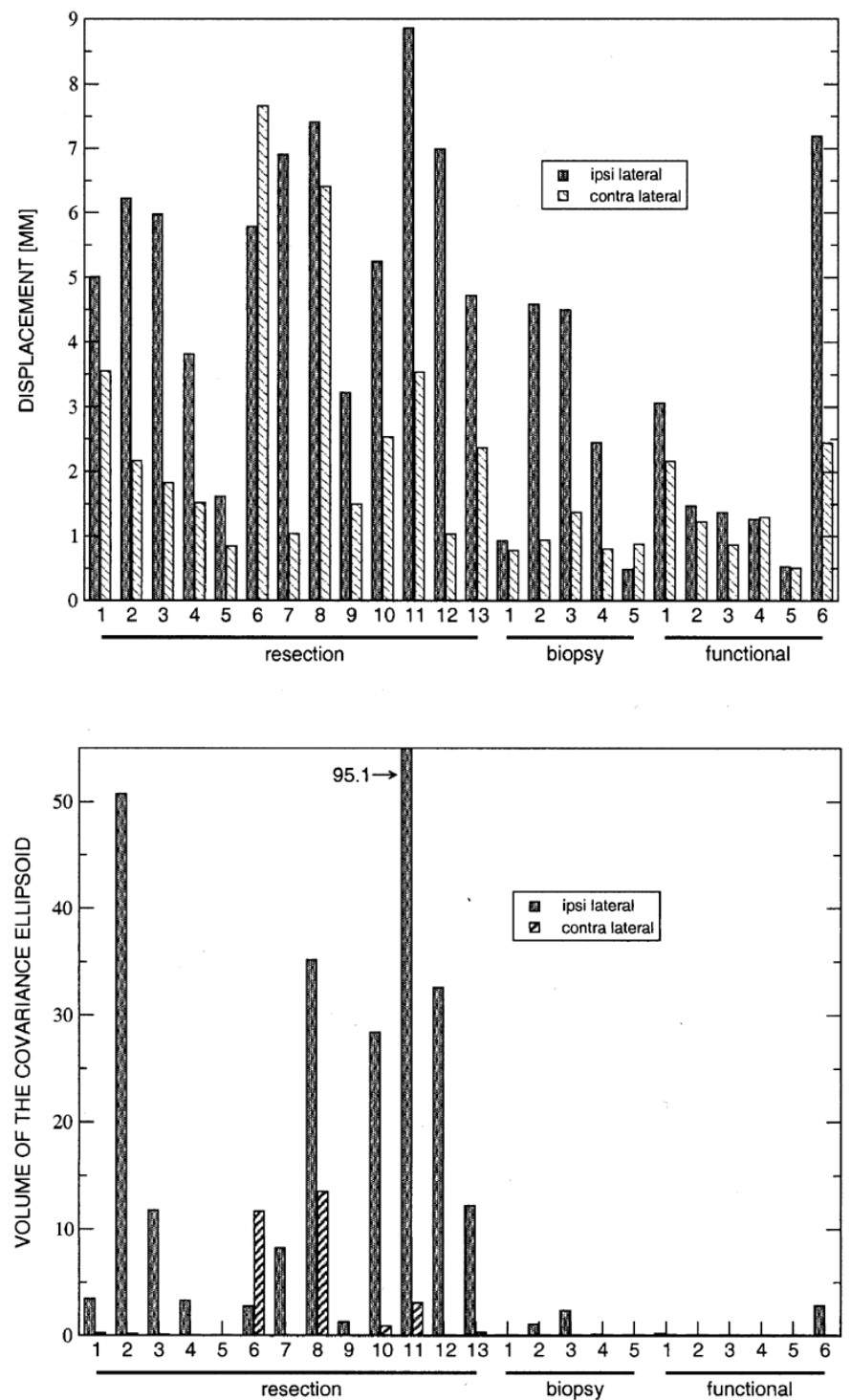

Fig. 8. Analysis of the displacement vectors in the ipsi- and contra-lateral hemisphere. (top) In order to ignore statistical outliers, we consider in the upper diagram the 95th percentile of the displacement magnitudes. (bottom) Degree of distribution of the displacement vector directions measured by the volume of the covariance ellipsoid based on the PCA of the deformation field (see Section II-C).

image (see Section II-D). The displacement vectors of the nonrigid algorithm are analyzed in two ways: First, the magnitude of the displacement field and the distribution of the displacement vectors determined by the PCA (see Section II-C) are investigated and illustrated in Fig. 8. Second, the volume change is determined for each voxel on the basis of the deformation field and the fifth percentile of the volume change-representing the maximal volume change-is depicted separately for each hemisphere in Fig. 9.

Comparing the magnitude of deformation for each hemispheres in Fig. 8 (top), in general, larger deformation occurs in the ipsi-lateral hemisphere than in the contra-lateral hemisphere. But the case resection6 also yields significant deformation values in the contra-lateral hemisphere. If you compare the resection cases with the biopsy and functional cases, it turns out, that the magnitude of displacement for the

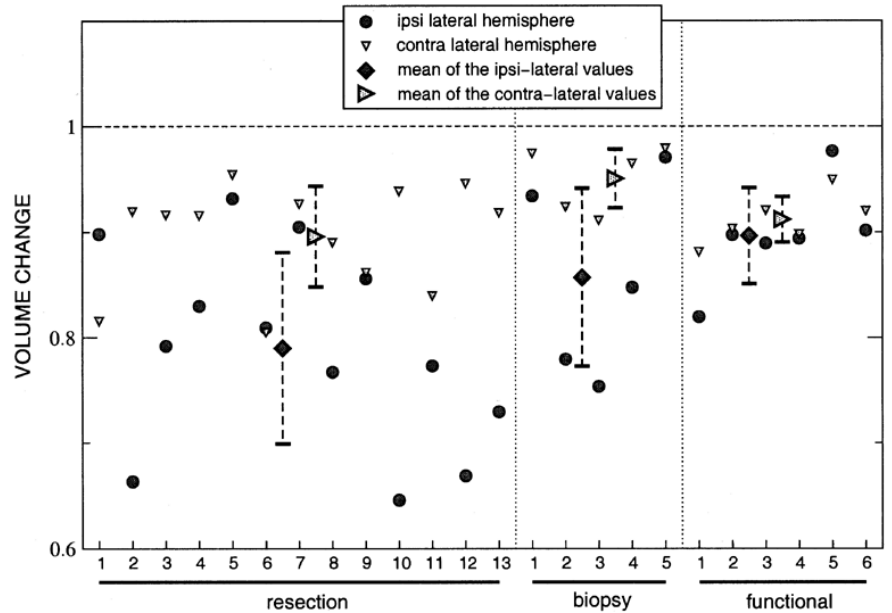

Fig. 9. Maximal volume change (shrinking) in the hemispheres. Since we are interested in the largest deformation values in each hemispheres, but not interested in statistic outliers, we consider the fifth percentile of the local volume change for each hemisphere. The value " 1 " represents no volume change and is marked with a dashed line in the diagram. Smaller values represent a shrinking, i.e., a volume change of the brain. The diamonds show for each type of intervention the mean of these cases in the ipsi-lateral hemisphere. The tilt triangles show the corresponding mean in the contra-lateral hemisphere. The adjacent dashed lines represent the standard deviations.

resection cases is, in general, larger. However, for instance, case functional6 shows a deformation with magnitude comparable to the magnitude of some resection cases. Fig. 8 (bottom) demonstrates that the direction of the displacement vectors in the resection cases can be much more distributed than in the biopsy and functional cases. In particular, whereas the deformation magnitude in case functional6 is substantially larger than, for instance, in case resection 13 , the direction of the displacement vectors is much less distributed. Since the magnitude of deformation is smaller in the contra-lateral hemisphere than in the ipsi-lateral hemisphere, the displacement directions are also less distributed in this hemisphere. However, in some cases with significant brain shift in the contra-lateral hemisphere the displacements occur in nearly the same direction, i.e., the distribution is nearly zero (for example, case resection 10 and resection 13 ).

Along with a larger displacement magnitude in the ipsi-lateral hemisphere, we also, in general, observe larger volume change in this hemisphere as illustrated in Fig. 9. However, the cases resection 1 and functional 5 show a larger shrinking in the contralateral hemisphere. Comparing the mean of the maximal volume change values separately for the resection, biopsy, and functional cases (compare the diamonds with each other), shows that, in general, the volume change in the resection cases is larger than in the biopsy cases, and the volume change in the biopsy cases is larger than in the functional cases. However, the standard deviation is relatively large in each group and, thus, the groups cannot be clearly separated.

\section{Volume Change of the Lateral Ventricle}

Fig. 10 summarizes, for each case, the volume change of the lateral ventricle determined by manual segmentation. The change is depicted for both ventricles together and for the ipsiand contra-lateral hemisphere separately. The variability in 


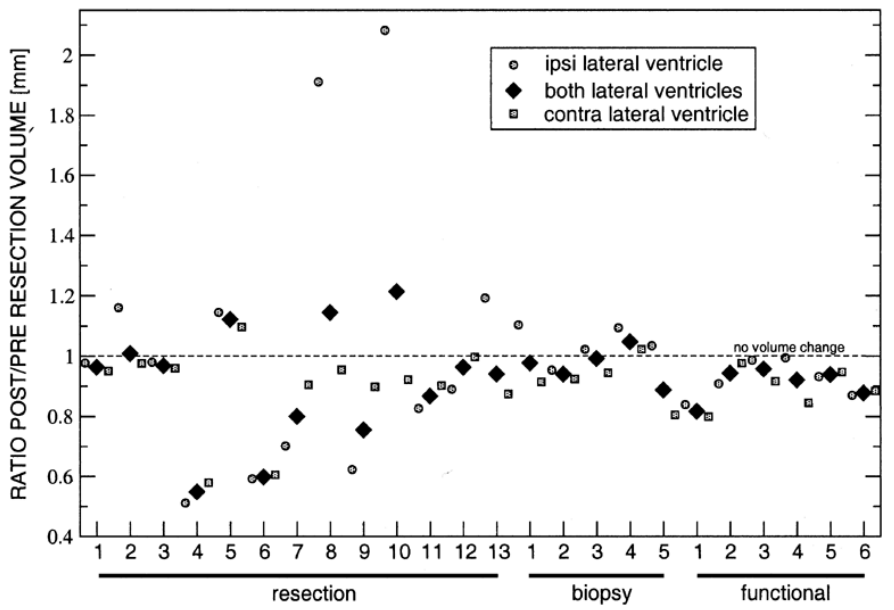

Fig. 10. Volume change of the lateral ventricle determined by manual segmentation. The $y$ axis represents the volume ratio of the lateral ventricle in the start- and end-procedure images, i.e., a value " 1 " represents no volume change, values larger than " 1 " represent an expansion, and values less than " 1 " represent a shrinking of the ventricle. The circles depict the volume change in the ventricle ipsi-lateral to the lesion, while the squares depict the volume change in the contra-lateral ventricle. The diamonds represent the volume change of both lateral ventricles together.

volume change measure assessed by repeated segmentation of a start- and end-procedure ventricle pair was 5.4\%.

Significant volume change is measured mainly in the resection cases, while both a shrinking (e.g., cases resection 4 and resection6) and an expansion (e.g., cases resections and resection 10) of the structure occurs. In general, the biopsy and functional cases show less volume change, but the ventricle also deforms significantly in some of these cases (e.g., case functional1). Comparing the volume change of the ipsi-lateral ventricle with the contra-lateral ventricle, shows that either the values are similar or the ipsi-lateral ventricle changes to a greater extent. The volume change in the ipsi-lateral ventricle varies between half (case resection4) and twice the original volume (case resection10).

\section{Deformation Values With Respect to Brain Surface Distance}

The distance map for each case described in Section II-D is used to analyze the displacement with respect to the distance to the brain surface in order to determine whether the shift is greatest at the brain surface. The displacement vector with the largest magnitude is selected for each distance and displayed in a diagram which produces scan lines shown in Fig. 11 (left). Analogously, the maximum and minimum volume changes - which represents the maximal expansion and maximal shrinking - are analyzed for each distance and visualized in Fig. 11 (right).

Comparison of the displacement diagrams in Fig. 11 shows that the location of the curve maximum, i.e., the maximal displacement in the whole brain, varies from case to case. Separating the distance from the brain surface to the center of the brain into five equal sized parts, reveals that for five cases the maximum is in the first fifth of this distance (i.e., close to the brain surface), for 13 cases it is in the second fifth, for four cases it is in the third fifth, and for two cases it is in the fourth fifth. In Fig. 11, one representative diagram for each group is depicted. These diagrams make clear that by the end of the procedure the main displacement does not only occur at or close to the brain surface, but also in deeper brain structures.

Comparing the displacement vector diagrams with the volume change diagrams, demonstrates that the position of the maximal volume change does not correspond with the position of the maximal displacement. For example, the maximal shrinking in resection 12 and resection 10 occurs in deeper brain structures than the maximal displacement. While resection 12 shows an expansion close to the brain surface, resection 10 shows no peak in the expansion curve. The large displacement in resection 7 close to the surface does not yield to a significant volume change, either. The case biopsy 4 has smaller displacement vectors but shows considerable shrinking.

\section{E. Relationship Between Displacement in the Hemisphere and Volume Change of the Lateral Ventricle}

Both the investigation of the displacement magnitudes and the volume change of the lateral ventricle show that brain structures in the ipsi-lateral hemisphere deform more than in the other hemisphere. In order to determine whether a significant brain shift is always associated with a volume change of the lateral ventricles, the largest displacement vector in one hemisphere is depicted in comparison to the manually determined volume change of the lateral ventricles (see Fig. 12).

The measurements of the contra-lateral hemisphere in the diagram are much more clustered than the measurements of the ipsi-lateral hemisphere. Since this cluster is close to "small displacement vectors" and "no volume change," this again indicates that the contra-lateral hemisphere deforms less, in general. In comparison with this the ipsi-lateral measurements are less clustered and in some cases, e.g., resection 10 and resection6, a large displacement is associated with a large volume change of the ventricle. However, in some other cases, e.g., resection 1 and biopsy3 large displacements were measured whereas almost no volume change occurred.

Altogether, these results suggest that a large displacement is not always associated with a volume change of the lateral ventricle.

\section{F. Direction of the Displacement Vectors}

The subtraction images in Fig. 5 suggest that the brain shift occurs mainly in the direction of gravity. In order to verify this observation, we compare the direction of gravity which is provided for each slice by the MR scanner with the direction of the first principal component of the displacement vectors (see Fig. 8), which can be considered as the main direction of the displacement field. The angle between these two vectors is depicted in Fig. 13 for 16 selected cases. In this diagram, cases are left out where magnitudes of deformation are so low, that the direction cannot be determined reliably (we omit cases which showed in Fig. 8 a maximal displacement less than $2 \mathrm{~mm}$ ).

Comparing the angle between these vectors shows that in some cases the main direction of the displacement field aligns well with the direction of gravity (e.g., case resection9, biopsy4, and functional1). In other cases, however, the brain shift occurs in a direction significantly different to that of gravity. For instance, in case resection 13 the main direction of the displacement field is nearly perpendicular to gravity. In Fig. 14, the ex- 


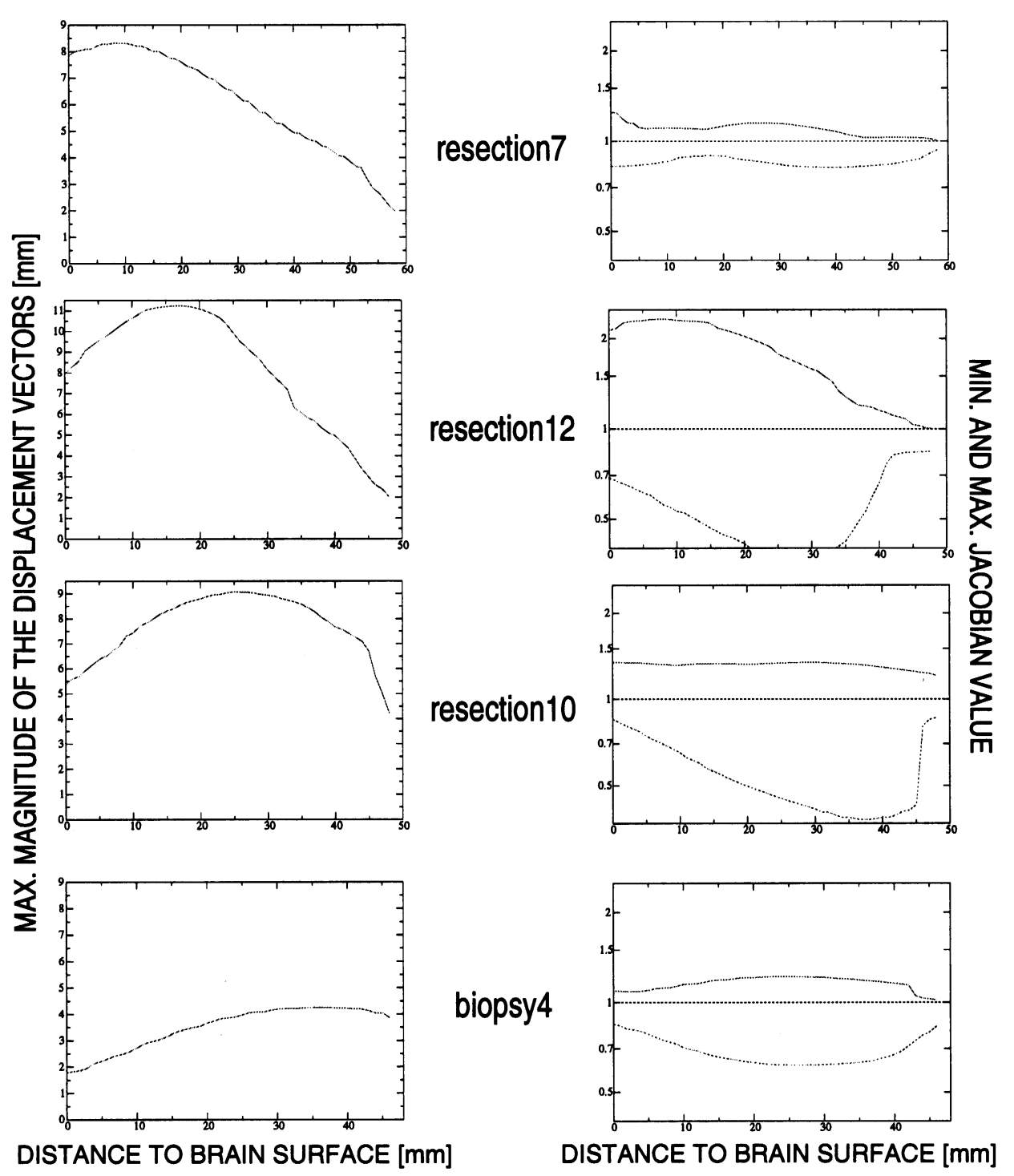

Fig. 11. Maximal displacement vector and local volume change with respect to the distance to the brain surface. (left) Maximal displacement vector with respect to the distance to the surface of the brain is depicted. (right) Analogously, the maximal local shrinking and expansion with respect to the distance to the surface is depicted. The value " 1 " (marked with a straight line in the diagrams) represents no volume change.

ample slice of resection13 suggests that because of the resected lesion a major part of the displacement vectors point in a different direction to the gravity vector and some even point in the opposite direction.

\section{DISCUSSION}

Twenty-four patients undergoing neurosurgery with intraoperative MR imaging were studied in order to quantify the interventional brain shift. The magnitude and location of deformation, and the distribution of the displacement directions were investigated using measurements determined by a nonrigid registration algorithm which provides displacement measurements throughout the brain. In addition, the volume change of the lateral ventricle was measured by manual segmentation. Careful study of brain deformation throughout the brain is important in ascertaining how easily computational algorithms could correct for brain deformation in IGS systems.
Our results are consistent with the results of previous studies (e.g., [17], [21], and [28]) and can be summarized as follows:

- Location of Deformation:

The manual segmentation of the lateral ventricles as well as the displacements determined by the nonrigid algorithm indicate that larger deformation occurs in the hemisphere ipsi-lateral to the lesion than in the contra-lateral hemisphere. These results agree well with previous studies [28]. However, some cases (both resection and no-resection cases) also show a significant brain shift in the contralateral hemisphere. Furthermore, considering the displacement scan-lines with respect to the brain surface distance, our study demonstrates that brain deformation does not only occur at the brain surface, but also in deeper brain structure. Even if the main displacement appears close to the surface, the resulting local volume change of the brain can occur further below the surface.

\section{- Magnitude of Deformation:}




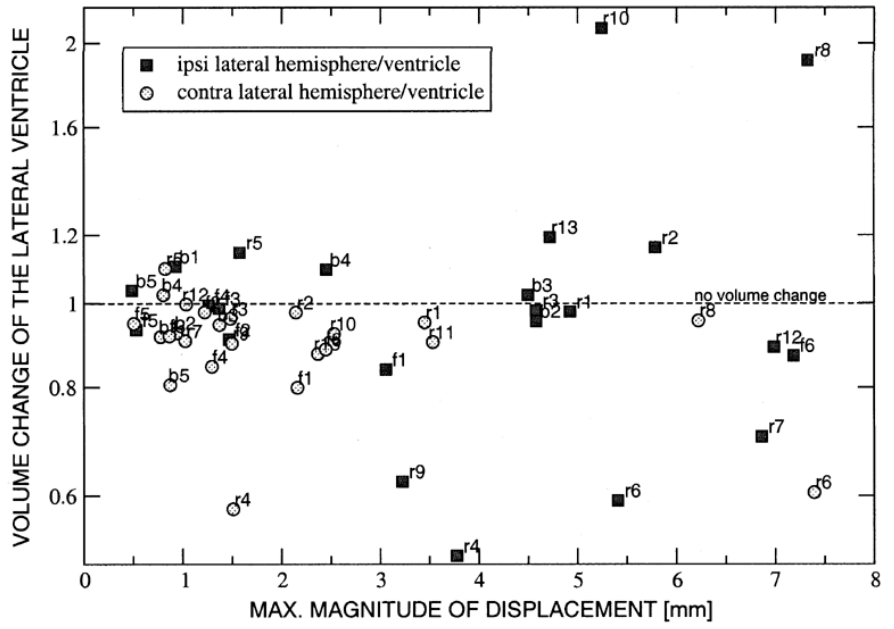

Fig. 12. Maximum displacement versus volume change of the lateral ventricle. The absolute value of the largest displacement is depicted on the $x$ axis of the diagram. The $y$ axis represents the volume change of the lateral ventricle, with the value "1" representing constant volume between the start- and end-procedure image. Each symbol represents the values of one case (marked for instance with $r l$ for case resection 1 ) separately for each hemisphere. The squares represent the values for the ipsi-lateral hemisphere and the circles represent the values for the contra-lateral hemisphere.

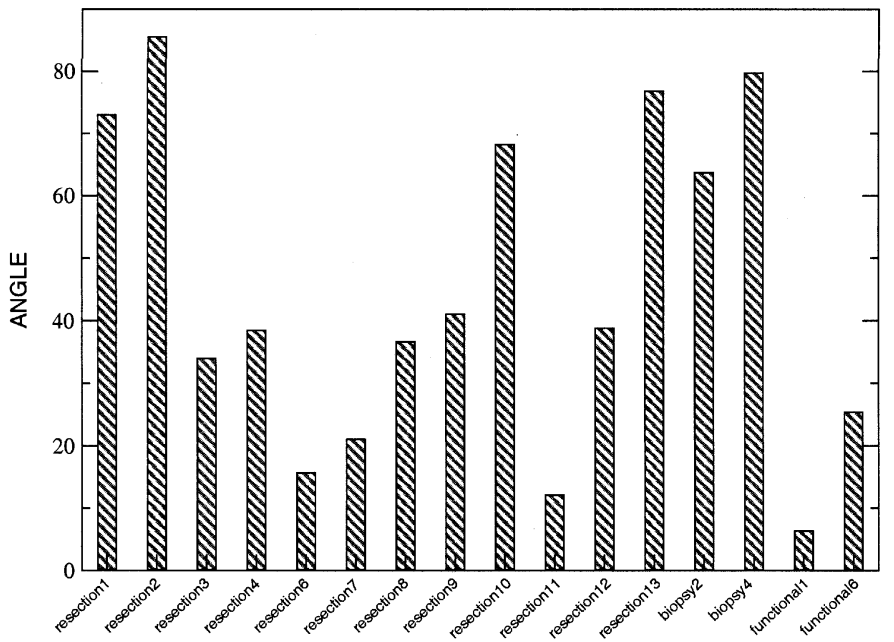

Fig. 13. Angle between the direction of gravity and the principal direction of the displacement field. Since a significant displacement is essential to reliably determine the direction of the displacement field, only cases with a significant displacement are shown. Cases whose maximal displacement (see Fig. 8) are less than $2 \mathrm{~mm}$ are ignored. For case biopsy3 no information about the direction of gravity was given and is, therefore, not considered.

We measured a shrinking of the lateral ventricles to up to half of the original volume and an expansion up to double the volume. In general, the magnitude of the displacement is larger in the resection cases in comparison with the functional or biopsy cases, but this varies significantly in each group.

- Predicting Deformation:

A simple view of the brain shift problem might be that the shift is caused by the brain collapsing under the force of gravity to fill space previously occupied by CSF and resected tissue. While gravity may be a major driving force in brain deformation, our results show that the

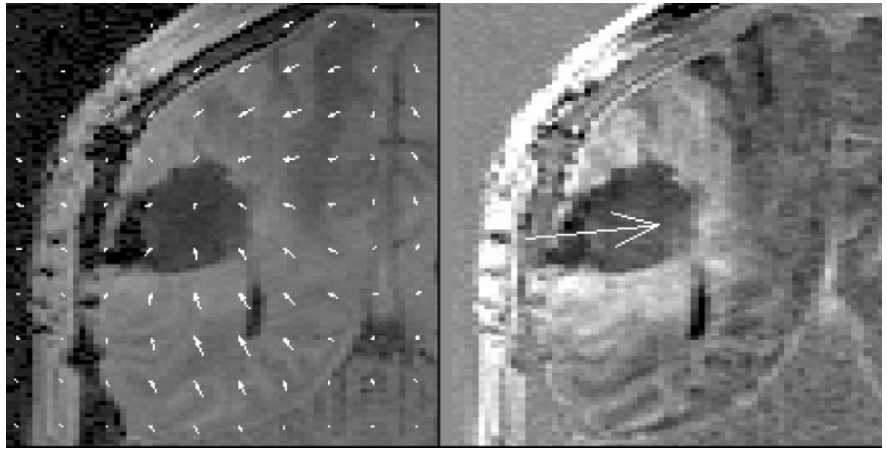

Fig. 14. Displacement field of case resection13. On the left side the end-procedure is overlaid with the nonrigid displacement field and on the right side the start/end-procedure subtraction image is depicted. The arrow indicates the direction of gravity in the images.

direction of gravity is a poor predictor of the direction of brain shift. In many subjects, the direction of the displacement field was not aligned with the direction of gravity (see, e.g., Fig. 14). The direction of shift is likely to be a consequence of a complex interplay between the force of gravity, boundary conditions (e.g., resected regions), fluid pressure, and other forces. Furthermore, substantial deformation is not always associated with substantial CSF loss from the lateral ventricles (Fig. 12), which suggests that measurement of CSF loss is not a good predictor of the magnitude of brain deformation. Thus, this investigation quantitatively confirms the comment in [17], that measuring only the volume change of the lateral ventricle is not reliable enough to quantify brain deformation.

Overall, the investigation demonstrates that though, in general, the deformation is less in the biopsy and functional than in the resection cases, significant brain shift can also occur in these cases (see, e.g., case functional6).

The pattern of brain deformation, both in terms of magnitude and direction of displacement, and of volume change, is extremely complex in this group of subjects. Therefore, simple computational algorithms that make use of very limited intraoperative information (e.g., brain surface shift) are unlikely to accurately predict brain deformation at the lesion for all cases. Simple biomechanical models might be able to predict brain deformation if they had sufficiently good boundary conditions. Our results suggest that it will be difficult to obtain these boundary conditions without using intraoperative imaging. For example, in some subjects, there is negligible deformation contra-lateral to the side of resection, but in other cases there is substantial deformation on both sides of the brain. An algorithm cannot, therefore, assume that the mid-line of the brain is fixed in all cases. Further research is needed in order to ascertain how well a biomechanical model needs to know mechanical properties of tissue and the boundary conditions in order to model the complex types of deformation we have observed in these subjects. Furthermore it is a mistake when using a traditional IGS system to assume that the brain shift at the surface is always a useful predictor of the amount of deformation further deep in the brain, or that the main direction of brain shift is in the direction of gravity. 


\section{ACKNOWLEDGMENT}

The authors would like to thank D. Rueckert, Department of Computing, Imperial College, London, for providing the nonrigid registration algorithm software used in this study.

\section{REFERENCES}

[1] H. Dickhaus, K. A. Ganser, A. Staubert, M. M. Bonsanto, C. R. Wirtz, V. M. Tronnier, and S. Kunze, "Quantification of brain shift effects by MR-imaging," in Proc. 19th Annu. Int. Conf. IEEE Engineering in Medicine and Biology Society, Chicago, IL, 1997, pp. 491-494.

[2] D. L. G. Hill, C. R. Maurer, R. J. Maciunas, J. A. Barwise, J. M. Fitzpatrick, and M. Y. Wang, "Measurement of intraoperative brain surface deformation under a craniotomy," Neurosurgery, vol. 43, no. 3, pp. 514-526, Sept. 1998

[3] N. L. Dorward, O. Alberti, B. Velani, F. A. Gerritsen, W. F. J. Harkness, N. D. Kitchen, and D. G. T. Thomas, "Postimaging brain distortion: Magnitude, correlates, and impact on neuronavigation," J. Neurosurg., vol. 88, pp. 565-662, April 1998.

[4] D. W. Roberts, A. Hartov, F. E. Kennedy, K. D. Paulsen, and M. I. Miga, "Intraoperative brain shift and deformation: A quantitative analysis of cortical displacement in 28 cases," Neurosurgery, vol. 32, no. 4, pp. 749-760, October 1998.

[5] C. R. Maurer, D. L. G. Hill, A. J. Martin, H. Liu, M. McCue, D. Rueckert, D. Lloret, W. A. Hall, R. E. Maxwell, D. J. Hawkes, and C. L. Truwit, "Investigation of intraoperative brain deformation using a 1.5 tesla interventional MR system: Preliminary results," IEEE Trans. Med. Imag., vol. 17, pp. 817-825, Oct. 1998

[6] G. J. Rubino, K. Farahani, and D. R. McGill, "Visualization of brain shift during interventional MRI-guided tumor removal," in Proc. Int. Society of Magnetic Resonance in Medicine (ISMRM), 1999, p. 509.

[7] R. B. Schwartz, L. Hsu, T. Z. Wong, D. F. Kacher, A. A. Zamani, P. M. Black, E. Alexander, III, P. E. Stieg, T. M. Moriarty, C. A. Martin, R. Kikinis, and F. A. Jolesz, "Intraoperative MR imaging guidance for intracranial neurosurgery: Experience with the first 200 cases," Neuroradiology, vol. 211, pp. 477-488, 1999.

[8] H. Iseki, H. Kawamura, T. Tanikawa, H. Kawabatake, T. Taira, K. Takakura, T. Dohi, and N. Hata, "An image-guided stereotactic system for neurosurgical operations," Stereotactic Functional Neurosurg., vol. 63, no. 1-4, pp. 130-138, 1994.

[9] R. D. Bucholz, D. D. Yeh, J. Trobaugh, L. L. McDurmont, C. D. Sturm, C. Baumann, J. M. Henderson, A. Levy, and P. Kessman, "Lecture Notes in Computer Sciences," in The correction of stereotactic inaccurancy caused by brain shift using an intraoperative ultrasound device, R. Mosges, J. Troccaz, and E. Grimson, Eds. Berlin, Germany: Springer-Verlag, 1997, vol. 1205, CVRMed MRCAS 97, pp. 459-466.

[10] K. D. Paulsen, M. I. Miga, F. E. Kennedy, P. J. Hoopes, A. Hartov, and D. W. Roberts, "A computational model for tracking subsurface tissue deformation during stereotactic neurosurgery," IEEE Trans. Biomed. Eng., vol. 46, pp. 213-225, Feb 1999.

[11] M. I. Miga, K. D. Paulsen, J. M. Lemery, S. Eisner, A. Hartov, F. E. Kennedy, and D. W. Roberts, "Model-updated image guidance: Initial clinical experience with gravity-induced brain deformation," IEEE Trans. Med. Imag., vol. 18, pp. 866-874, Oct. 1999.

[12] M. I. Miga, K. D. Paulsen, F. E. Kennedy, P. J. Hoopes, A. Hartov, and D. W. Roberts, "In vivo analysis of heterogeneous brain deformation computations for model-updated image guidance," Comput. Meth. Biomech. Biomed. Eng., vol. 3, no. 2, pp. 129-146, 2000.

[13] _ - "In vivo quantification of a homogeneous brain deformation model for updating preoperative images during surgery," IEEE Trans. Biomed. Eng., vol. 47, pp. 266-273, Feb. 2000

[14] M. I. Miga, D. W. Roberts, F. E. Kennedy, L. A. Platenik, A. Hartov, K. E. Lunn, and K. D. Paulsen, "Modeling of retraction and resection for intraoperative updating of images," Neurosurgery, vol. 49, no. 1, pp. 75-84, July 2001

[15] A. D. Castellano-Smith, T. Hartkens, J. Schnabel, D. R. Hose, H. Lui, W. A. Hall, C. L. Truwit, D. J. Hawkes, and D. L. G. Hill, "Constructing patient specific models for correcting intraoperative brain deformation," in Lecture Notes in Computer Science, W. J. Niessen and M. A. Viergever, Eds. Berlin, Germany: Springer-Verlag, 2001, vol. 2208, Conf. Medical Image Computing and Computer-Assisted Intervention (MICCAI '01), pp. 1091-1098.
[16] P. J. Kelly, B. A. Kall, S. Goerss, and F. Earnest, IV, "Computer-assisted stereotaxic laser resection of intra-axial brain neoplasms," J. Neurosurg., vol. 64, pp. 427-439, Mar. 1986.

[17] A. Nabavi, P. McL. Black, D. T. Gering, C.-F. Westin, V. Mehta, R. S. Pergolizzi, M. Ferrant, S. K. Warfield, N. Hata, R. B. Schwartz, W. M. Wells, III, R. Kikinis, and F. A. Jolesz, "Serial intraoperative magnetic resonance imaging of brain shift," Neurosurgery, vol. 48, no. 4, pp. 787-798, April 2001.

[18] P. M. Thompson, J. N. Giedd, R. P. Woods, D. MacDonald, A. C. Evans, and A. W. Toga, "Growth patterns in the developing brain detected by using continuum mechanical tensor maps," Nature, vol. 404, no. 9, pp. 190-193, Mar. 2000.

[19] W. R. Crum, A. Freeborough, and N. C. Fox, "The use of regional fast fluid registration of serial MRI to quantify local change in neurodegenerative disease," in Medical Image Understanding and Analysis, D. Hawkes, D. Hill, and R. Gaston, Eds. Oxford, U.K.: Spinger, July 1999 , pp. 25-28.

[20] W. R. Crum, R. I. Scahill, and N. C. Fox, "Automated hippocampal segmentation by regional fluid registration of serial MRI: Validation and application in Alzheimer's disease," Neuroimage, vol. 13, pp. 847-855, 2001.

[21] N. Hata, A. Nabavi, W. Wells, III, S. K. Warfield, R. Kikinis, P. McL. Black, and F. A. Jolesz, "Three-dimensional optical flow method for measurement of volumetric brain deformation from intraoperative MR images," J. Comput. Assist. Tomogr., vol. 24, no. 4, pp. 531-538, 2000.

[22] N. Hata, A. Nabavi, S. Warfield, W. Wells, R. Kikinis, and F. A. Jolesz, "A volumetric optical flow method for measurement of brain deformation from intraoperative magnetic resonance images," in MICCAI'99, Cambridge, U.K., 1999, pp. 928-935.

[23] A. Nabavi, D. Gering, M. Ferrant, M. Kaus, D. Kacher, R. S. Pergolizzi, V. Mehta, W. Wells, S. Timoner, C. T. Mamisch, M. Das, S. Bonitz, N. Hata, S. Warfield, C. F. Westin, P. Everet, P. E. Stieg, R. Kikinis, W. E. L. Grimson, P. McL. Black, and F. Jolesz, "'Brain shift' in the intraoperative MRI," in Proc. Int. Society of Magnetic Resonance (ISMRM), 2000, p. 1329.

[24] D. L. G. Hill, C. R. Maurer, C. Studholme, J. M. Fitzpatrick, and D. J. Hawkes, "Correcting scaling errors in tomographic images using a nine degree of freedom registration algorithm," J. Comput. Assist. Tomogr., vol. 22, pp. 317-323, 1998.

[25] C. Studholme, D. L. G. Hill, and D. J. Hawkes, "An overlap invariant entropy measure of 3D medical image alignment," Pattern Recogn., vol. 32, pp. 71-86, 1999.

[26] D. Rueckert, L. I. Sonoda, C. Hayes, D. L. G. Hill, M. O. Leach, and D. J. Hawkes, "Non-rigid registration using free-form deformations: Application to breast MR images," IEEE Trans. Med. Imag., pp. 712-721, Aug. 1999.

[27] E. R. E. Denton, L. I. Sonoda, D. Rueckert, S. C. Rankin, C. Hayes, M. Leach, D. L. G. Hill, and D. J. Hawkes, "Comparison and evaluation of rigid and nonrigid registration of breast MR images," JCAT, vol. 23, pp. 800-805, 1999.

[28] D. L. G. Hill, C. R. Maurer, A. J. Martin, S. Sabanathan, W. A. Hall, D. J. Hawkes, D. Rueckert, and C. L. Truwit, "Assessment of intraoperative brain deformation using interventional MR imaging," in Lecture Notes in Computer Science, C. Taylor and A. Colchester, Eds. Cambridge, UK: Springer, Sept. 1999, vol. 1679, MICCAI'99, pp. 910-919.

[29] D. Rey, G. Subsol, H. Delingette, and N. Ayache, "Automatic detection and segmentation of evolving processes in 3D medical images: Application to multiple sclerosis," in Lecture Notes in Computer Science, A. Kuba et al., Eds. Visegrád, Hungary: Springer, 1999, vol. 1613, IPMI'99, pp. 154-167.

[30] C. Davatzikos, "Spatial normalization of three-dimensional brain images using deformable models," J. Comput. Assist. Tomogr, vol. 20, no. 4, pp. 656-665, July-Aug. 1996.

[31] Massachusetts General Hospital. Internet Brain Segmentation Repository (IBSR). [Online]. Available: http://neuro-www.mgh.harvard.edu/cma/ibsr.

[32] C. R. Maurer, R. Qi, and V. Raghavan, "A linear time algorithm for computing exact Euclidean distance transforms of binary images in arbitrary dimensions," IEEE Trans. Pattern Anal. Machine Intell., vol. 25, pp. 265-270, Feb. 2003. 